\title{
CONTROLE QUÍMICO E BIOLÓGICO DA BROCA-PELUDA, Hyponeuma sp. (LEPIDOPTERA: NOCTUIDAE), NA CANA-DE-AÇÚCAR
}

RODRIGUES, Alex Danilo ${ }^{1}$

PINTO, Alexandre de Sene ${ }^{2}$ SEGATO, Silvelena Vanzolini ${ }^{3}$

RESUMO: A cana-de-açúcar é uma cultura que vem se expandindo gradativamente e vem mostrando grande potencial econômico. Devido às diversas condições climáticas e demais fatores, muitas lavouras estão sendo infestadas por novas pragas. Uma das mais recentes é a broca-peluda, Hyponeuma taltula (Schaus) (Lepidoptera: Noctuidae), com poucas informações geradas e sem medidas de controle até o momento.O presente estudo teve por objetivo avaliar a eficácia de inseticidas e fungos entomopatogênicos no controle da broca-peluda em cana-deaçúcar, em Ribeirão Preto, SP. O ensaio foi instalado na variedade SP 81-3250, cana-soca, em um delineamento em blocos casualizados, onde cinco tratamentos foram repetidos quatro vezes, em parcelas de $42 \mathrm{~m}^{2}$. Os tratamentos foram: (i) Beauveria bassiana (Boveriz ${ }^{\circledR}$ Biocontrol, $5 \times 10^{13}$ conídios ha ${ }^{-1}$ ); (ii) Metarhizium anisopliae (Metarriz ${ }^{\circledR}$ Biocontrol, $5 \times 10^{13}$ conídios ha ${ }^{-1}$ ); (iii) carbofurano (Furadan ${ }^{\circledR} 350 \mathrm{SC}, 5 \mathrm{~L} \mathrm{ha}^{-1}$ ); (iv) tiametoxam (Actara ${ }^{\circledR}$ $250 \mathrm{WG}, 1,5 \mathrm{Kg} \mathrm{ha}^{-1}$ ); (v) testemunha. As avaliações foram periódicas, contando-se o número de lagartas vivas em um metro linear por repetição. Os fungos testados não foram eficazes no controle da broca-peluda, provavelmente por que foram aplicados em período seco. Os inseticidas carbofurano e tiametoxam foram eficientes no controle da praga a partir dos 14 dias após a aplicação, mas tiametoxam causou redução de 93,4\% aos 28 dias após a aplicação, sendo esse o inseticida mais eficiente.

Palavras-chave: Praga agrícola. Controle químico Controle microbiano.

\section{CHEMICAL AND BIOLOGICAL CONTROL OF THE HYPONEUMASP. (LEPIDOPTERA: NOCTUIDAE) IN SUGARCANE}

SUMMARY: The sugarcane is a crop that has been expanding gradually and is showing great economic potential. Due to various weather conditions and other factors, many crops are infested by new pests. One of the most recent is Hyponeuma taltula (Schaus) (Lepidoptera: Noctuidae), with little information generated and without control measures so far. This study aimed to evaluate the effectiveness of insecticides and entomopathogenic fungi to control Hyponeuma sp. in sugarcane, in Ribeirão Preto, São Paulo state. The experiment was carried out in variety SP 813250 , ratoon cane, in a randomized block design, where five treatments were repeated four times in plots of $42 \mathrm{~m}^{2}$. The treatments were: (i) Beauveria bassiana (Boveriz ${ }^{\mathrm{TM}}$ Biocontrol, $5 \times 10^{13}$ conidia ha $^{-1}$ ), (ii) Metarhizium anisopliae (Metarriz ${ }^{\mathrm{TM}}$ Biocontrol, 5x10 13 conidia ha ${ }^{-1}$ ), (iii) carbofuran (Furadan ${ }^{\mathrm{TM}} \mathrm{SC} 350,5 \mathrm{~L} \mathrm{ha}^{-1}$ ), (iv) thiamethoxam (Actara ${ }^{\text {TMWG }}$ 250, $1.5 \mathrm{~kg} \mathrm{ha}^{-1}$ ), (v) control. The evaluations were periodic, counting the number of live caterpillars per linear meter per replicate. The fungi tested were not effective in controlling Hyponeuma sp., probably because they were applied in the dry season. The insecticides carbofuran and thiamethoxam were efficient in controlling the pest from 14 days after application, but thiamethoxam caused a reduction of $93.4 \%$ at 28 days after application, which is the most effective insecticide.

Keywords: Crop pest. Chemical control. Microbial control.

\section{INTRODUÇÃO}

O Brasil é o maior produtor de cana-de-açúcar do mundo, sendo que na safra 2010/2011 atingiu 8,1 milhões de hectares plantados e 624,5 milhões de toneladas produzidas (produtividade média de 77,7 t

\footnotetext{
${ }^{1}$ Aluno de graduação da Instituição Universitária Moura Lacerda;

${ }^{2}$ Professor da Instituição Universitária Moura Lacerda. aspinn@ @uol.com.br;

${ }^{3}$ Professora da Instituição Universitária Moura Lacerda. sv.segato@ bol.com.br.
} 
$\mathrm{ha}^{-1}$ ) (UDOP, 2011). A cana-de-açúcar é um agro ecossistema que abriga numerosas espécies de insetos, sendo que algumas delas, dependendo da época do ano e da região, podem ocasionar sérios prejuízos econômicos (PARRA; BOTELHO; PINTO, 2010).

No Brasil, várias são as pragas relacionadas à cultura da cana-de-açúcar. Silva et al. (1968) relacionaram 111 insetos hóspedes desta cultura, dos quais 32 são lepidópteros. Alguns desses lepidópteros são bem conhecidos pelos agricultores, que são a broca-da-cana-de-açúcar, Diatraea saccharalis(Fabricius) (Crambidae), broca-gigante, Telchin licus(Drury) (Castnidae) e lagarta-elasmo, Elasmopalpus lignosellus(Zeller) (Pyralidae).

Entretanto, na década de 1970 alguns relatos de ocorrência de uma lepidobroca, em Santa Catarina, atacando a cana-de-açúcar distinguiram uma nova praga. Guagliumi (1973) comentou que essa praga era um lepidóptero distinto dos demais, danificando os colmos como Metamasius sp. (Coleoptera: Curculionidae). Guagliumi e Mendes (1974) identificam esse lepidóptero como Hyponeuma taltula (Schaus) (Lepidoptera: Noctuidae), danificando colmos também em São Paulo e Pernambuco. Desde então, quase nada foi publicado sobre essa praga, restringindo-se a uma nota sobre parâmetros biológicos e imagens das larvas, pupas e adultos (PLANALSUCAR, 1974), caracterização morfológica de imaturos (ZENKERet al., 2007) e imagens do ciclo de vida desse inseto, que ficou conhecido como broca-peluda (PINTO; BOTELHO; OLIVEIRA, 2009).

A broca-da-cana-de-açúcar e a cigarrinha-das-raízes, Mahanarva fimbriolata Stål (Hemiptera: Cercopidae), são as principais pragas dos canaviais do país (PINTO; BOTELHO; OLIVEIRA, 2009). Essas duas pragas são responsáveis por mais de $40 \%$ de perdas na produção de cana-de-açúcar, quando presentes e não controladas (PINTO; GARCIA; OLIVEIRA, 2006).

Os produtos registrados para o controle de M. fimbriolata na cultura da cana-de-açúcar no Brasil são: aldicarbe e carbofurano (carbamato), etiprole (pirazol), imidacloprido (neonicotinoide), lambdacialotrina + tiametoxam (pitretroide + neonicotinoide), Metarhizium anisopliae (produto biológico) e tiametoxam (neonicotinoide) (AGROFIT, 2011b). Para D. saccharalis são:Bacillus thuringiensis Berliner, Cotesia flavipes (Cam.) (Hymenoptera: Braconidae) e Trichogramma galloi Zucchi (Hymenoptera: Trichogrammatidae) (produtos biológicos), carbofurano (carbamato), clorantraniliprole (antranilamida), fipronil (pirazol), lambda-cialotrina + tiametoxam (piretroide + neonicotinoide), lufenurom, novalurom e triflumuron (benzoilureia) e tebufenozida (diacilhidrazina) (AGROFIT, 2011a).

Apesar de não registrado para a cultura, o fungo Beauveria bassiana é citado como importante controlador de diversas pragas da cana-de-açúcar, como a broca-da-cana (MENDONÇA et al., 1996; ALVES, 1998; SILVEIRA et al., 2007), o gorgulho-da-cana, Sphenophorus levis (Coleoptera: Curculionidae) (BADILLA; ALVES, 1991; MACEDO; GARCIA; BOTELHO, 2006), e os cupins (ALMEIDA, 1994; ALMEIDA; ALVES, 1996; ALMEIDA; ALVES, 1999).

Dessa forma, sem informações sobre o controle dessa nova praga e conhecendo os produtos biológicos e químicos registrados para a cultura ou com potencial de uso, esse trabalho teve por objetivo avaliar a eficácia de fungos entomopatogênicos e de inseticidas no controle da broca-peluda em canavial.

\section{MATERIAL E MÉTODOS}

O experimento foi conduzido em campo, na propriedade do Sr. Luís Carlos Rodrigues, no Sítio Nossa Senhora da Aparecida, localizado no município de Ribeirão Preto, SP. As coordenadas geográficas aproximadas são de $21^{\circ} 15^{\prime}$ de latitude sul e $47^{\circ} 52^{\prime}$ de longitude oeste de Greenwich, com altitude aproximada de $743 \mathrm{~m}$.O clima da região, segundo a classificação de Köppen é Tropical Cfb. A temperatura média anual é de $21,5^{\circ} \mathrm{C}$. 
A variedade de cana-de-açúcar utilizada foi a SP 81-3250, com três meses de desenvolvimento, segundo corte. O delineamento experimental constituiu-se de blocos casualizados, com cinco tratamentos e quatro repetições cada um. Cada parcela foi de $42 \mathrm{~m}^{2}(20 \times 20 \mathrm{~m})$, compreendendo três linhas de $10 \mathrm{~m}$ lineares cada, com espaçamento entre linhas de 1,4 m. Os produtos químicos foram aplicados em um volume de $150 \mathrm{~L} \mathrm{ha}^{-1}$ de calda.

Os tratamentos testados foram: (1) fungo Beauveria bassiana (Boveriz ${ }^{\circledR}$ Biocontrol), no equivalente a $5 \times 10^{13}$ conídios viáveis $\mathrm{ha}^{-1}$ (5 Kg arroz + conídios); (2) fungo Metarhizium anisopliae (Metarriz ${ }^{\circledR}$ Biocontrol), no equivalente a $5 \times 10^{13}$ conídios viáveis ha ${ }^{-1}(5 \mathrm{Kg}$ arroz + conídios);(3)inseticida carbamato carbofurano (Furadan ${ }^{\circledR} 350 \mathrm{SC}$ ), no equivalente a $5 \mathrm{~L} \mathrm{ha}^{-1}$;(4)inseticida neonicotinoide tiametoxam (Actara ${ }^{\circledR} 250 \mathrm{WG}$ ), no equivalente a $1,5 \mathrm{Kg} \mathrm{ha}^{-1}$;(5)testemunha (sem aplicação de quaisquer inseticidas).

Para a aplicação dos produtos químicos foi utilizado um implemento agrícola (aplicador de inseticida em soqueiras) acoplado a um trator, para permitir que o produto fosse enterrado (aplicado) diretamente na linha da cana de açúcar. Tal equipamento permitiu que o produto fosse aplicado em três linhas de soqueiras, simultaneamente. Cada conjunto possuía um disco de corte de 26 polegadas com molas de compressão que fizeram o corte do colchão de palha. Fixado logo atrás do disco de corte, havia um dispositivo com bico de pulverização que fazia a aplicação do produto em profundidade (de 5 a 20 $\mathrm{cm})$. O tanque do implemento possuía capacidade para $600 \mathrm{~L}$ de calda, o que acelerou a aplicação dos produtos.

Para a aplicação dos produtos biológicos, o método escolhido foi o manual, utilizando-se de uma bomba costal, com capacidade para $5 \mathrm{~L}$ de calda. Este método permitiu a aplicação do produto diretamente sobre a soqueira. A pulverização foi realizada no final da tarde, para se evitar a insolação.

As avaliações foram realizadas periodicamente, contando-se o número de lagartas encontradas por metro linear de cada parcela.

Todos os dados obtidos foram submetidos à análise de variância (ANOVA). Quando o teste $\mathrm{F}$ da ANOVA indicou significância de 5\% de probabilidade de erro, procederam-se as análises complementares por meio do teste de Tukey a $5 \%$ de probabilidade, onde as médias foram comparadas. Todos os cálculos estatísticos foram realizados pelo programa Statistica for Windows (STATSOFT, 1996).

Para facilitar a compreensão da real ação dos inseticidas e fungos testados, calculou-se a porcentagem média de mortalidade de lagartas para cada produto e fez-se a correção (\% RC) desse valor pela fórmula de Henderson e Tilton (1995), que leva em consideração a população para cada tratamento antes e após a aplicação dos mesmos.

\section{RESULTADOS E DISCUSSÃO}

No momento de instalação do ensaio, a população de Hyponeuma sp. no local do experimento apresentava 14,95 \pm 0,50 lagartas por metro linear, em média, sem diferenças estatísticas entre os tratamentos (Figura 1).

Após 7 dias da aplicação dos produtos (10/10/2011), não houve diferenças significativas entre os tratamentos (Figura 1).

Entretanto, aos 14 dias após (17/10), os tratamentos carbofurano e tiametoxam não diferiram entre si, mas estatisticamente foram diferentes dos demais tratamentos, que apresentaram os maiores valores de lagartas por metro (Figura 1).

Em 24/10, 21 dias após a pulverização, os tratamentos apresentaram as mesmas diferenças. 
Na última avaliação, aos 28 dias após a aplicação (31/10), o tratamento tiametoxam apresentou menos do que uma lagarta viva por metro, em média, diferindo significativamente do tratamento carbofurano, que apresentou média de 5,5 lagartas por metro, que por sua vez diferiu dos demais tratamentos, com os maiores valores (Figura 1).

Figura 1.Número médio de lagartas de Hyponeuma sp. por metro linear em várias datas antes e após a aplicação de inseticidas e fungos entomopatogênicos. Ribeirão Preto, SP, 2011. Pontos seguidos pela mesma letra não diferem entre si pelo teste de Tukey $(\mathrm{p}>0,05)$. A seta indica a data de aplicação dos produtos.

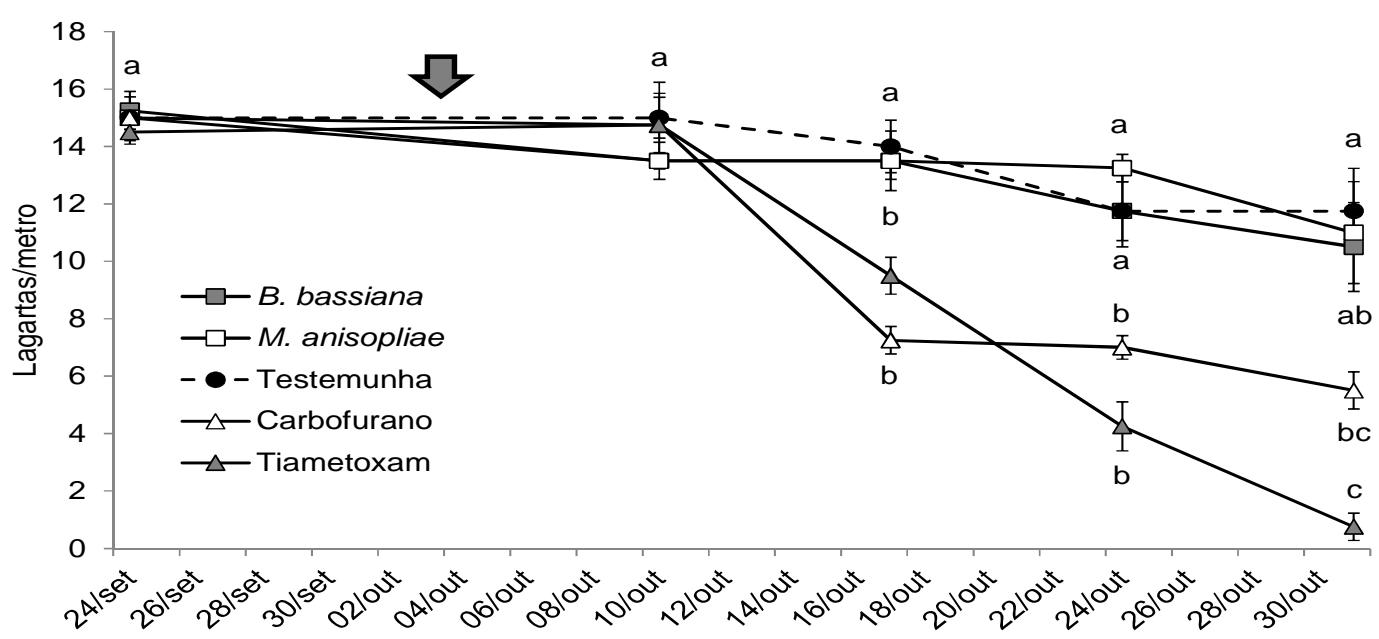

Calculando a porcentagem média de redução populacional de Hyponeuma sp. e procedendo a correção pela fórmula de Henderson e Tilton (1955), pôde-se verificar que aos 7 dias após a aplicação dos produtos, nenhum deles foi eficiente na redução da praga (Figura 2).

A eficácia dos inseticidas começa a se tornar evidente aos 14 dias após a aplicação, quando carbofurano reduziu, em média, 48,2\% e tiametoxam, 29,8\% da quantidade de lagartas. Os fungos não atingiram $10 \%$ de controle (Figura 2).

Aos 21 dias da aplicação, o inseticida tiametoxam ultrapassa a eficácia de carbofurano, chegando a $62,6 \%$ de redução média, contra $40,4 \%$ de carbofurano. A eficácia de controle dos fungos ainda continuou muito baixa (Figura 2).

No final do ensaio, aos 28 dias após a pulverização, tiametoxam confirma a maior eficácia de controle, atingindo a média de 93,4\% de redução de lagartas de Hyponeuma sp., enquanto que carbofurano chegou a 53,2\% e os fungos Beauveria bassiana, 12,1\%, e Metarhizium anisopliae, 6,4\% (Figura 2).

Os dois inseticidas testados controlaram as lagartas de Hyponeuma sp., mas tiametoxam foi o produto mais eficiente, até os 28 dias após a aplicação. Como carbofurano está sendo reavaliado no Brasil quanto à autorização de seu uso (OECO, 2011), a única alternativa é o produto tiametoxam. 
Figura 2. Porcentagem média de redução corrigida (HENDERSON; TILTON, 1955) de lagartas de Hyponeuma sp. após a aplicação de inseticidas e fungos entomopatogênicos. Ribeirão Preto, SP, 2011.

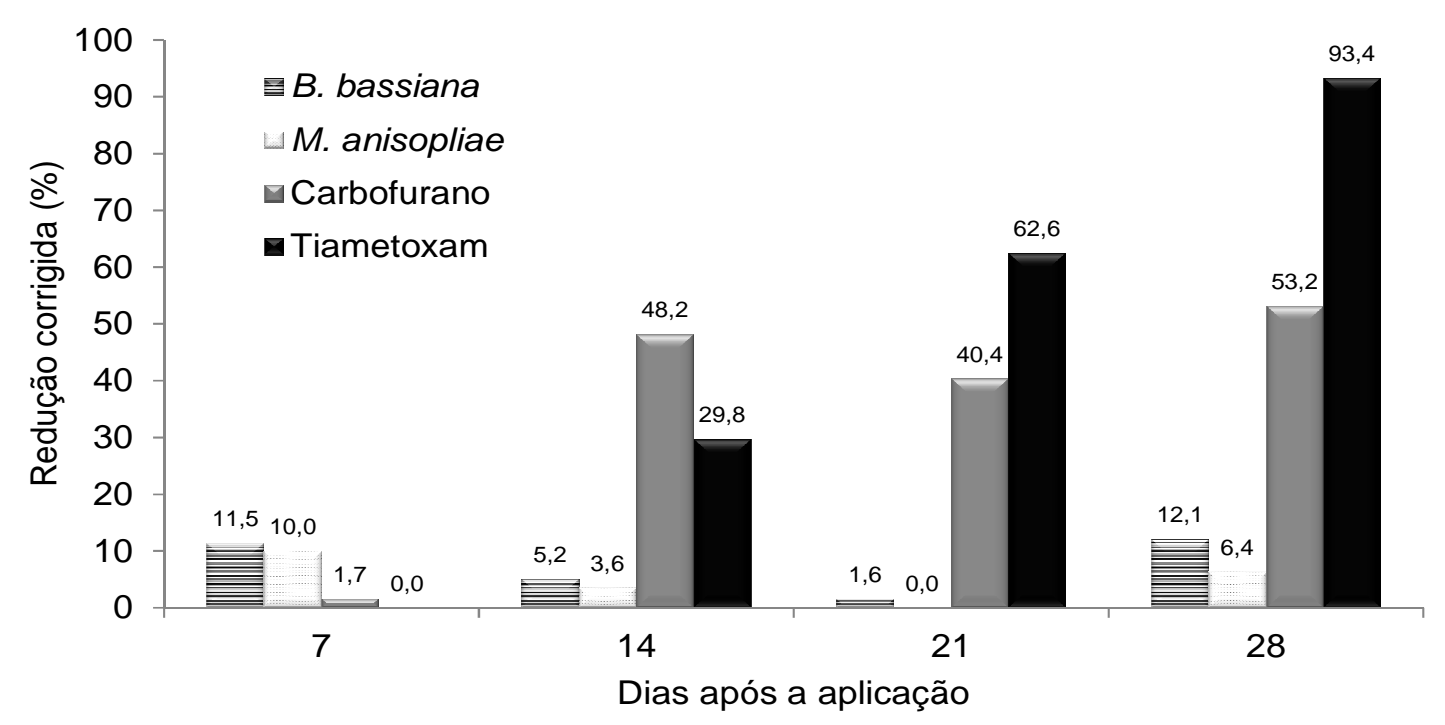

Os resultados obtidos com os inseticidas concordam com aqueles de Levine e Felsot (1985) e Azeredo e Cassino (2010), para Agrotis ipsilon, e Wang e Dauterman (1995), para Helicoverpa zea. Mas discordam de Martins et al. (2006), que verificaram que tiametoxam não foi eficiente no controle de Spodoptera frugiperda no tratamento de sementes.

Entretanto, o desempenho dos fungos do atual ensaio discorda de quase todos os trabalhos citados para M. anisopliae (GUTIÉRREZ; CARDENAS; GUTIÉRREZ, 1995; PINTO; ANNIBAL; ANIBAL, 2004; RONDA, 2004; FARAG, 2008; KULKARNI et al., 2008; CANINI, 2010; MARTINS, 2010) e B. bassiana (CASTANHEIRA et al., 1993; FARAG, 2008; KAUR; PADMAJA, 2008; MICHEREFF et al., 2008; CANINI, 2010;MARTINS, 2010), que obtiveram resultados satisfatórios no controle de diversas espécies de noctuídeos.

Entretanto, Gosselinet et al. (2009), Vercesi et al. (2008), Danieli et al. (2009) e Afonso Jr. et al. (2009) não obtiveram bons resultados de eficiência dos fungos mencionados. Nos três últimos trabalhos, os autores acreditaram que a não eficiência estava ligada a baixa infestação de lagartas de Spodoptera frugiperda em milho, o que não foi o caso no atual o ensaio. Mas também acreditaram que a aplicação dos fungos em épocas desfavoráveis (baixa umidade ou temperatura) afetou o desempenho dos mesmos, o que pode ter acontecido nesse experimento. Os fungos foram aplicados em um período seco, anterior ao início das chuvas, que tardaram a iniciar. Além disso, os fungos foram aplicados sobre as soqueiras, o que pode ter influenciado negativamente o controle.

Novos ensaios deverão ser conduzidos com os fungos e com outros inseticidas para serem testadas novas opções de controle da broca-peluda. Entretanto, com os resultados obtidos, uma alternativa de controle surge para os agricultores tão carentes de informações.

\section{CONCLUSÃO}

Baseado nas condições em que o ensaio foi conduzido, em período com baixa umidade relativa do ar e ausência de precipitação pluviométrica, pode-se concluir que:

- os inseticidas carbofurano e tiametoxam são eficientes no controle da broca-peluda, Hyponeuma sp. (Lepidoptera: Noctuidae), até 28 dias após a aplicação;

- o inseticida tiametoxam é o inseticida mais eficiente no controle da broca-peluda; 
- os fungos Beauveria bassiana e Metarhizium anisopliae, aplicados sobre a soqueira, não são eficientes no controle da broca-peluda.

\section{REFERÊNCIAS}

AFONSO JUNIOR., V.A. et al. Eficiência dos fungos Metarhizium anisopliaee Beauveria bassiana no controle de Spodoptera frugiperda em milho "safrinha". In: SIMPÓSIO DE CONTROLE BIOLÓGICO, 11., Bento Gonçalves, 2009. Resumo... Porto Alegre: Unisinos, 2009. (CD-ROM)

AGROFIT - Sistema de Agrotóxicos Fitossanitários. Disponível em:

$<$ http://bi.agricultura.gov.br/reports/rwservlet?agrofit_cons\&pragas.rdf\&p_script_body=\&p_id_cultura_prag a=2806\&paramform=no> Acesso em: 11 nov.2011a.

AGROFIT - Sistema de Agrotóxicos Fitossanitários. Disponível em:

$<$ http://bi.agricultura.gov.br/reports/rwservlet?agrofit_cons\&pragas.rdf\&p_script_body=\&p_id_cultura_prag a=2816\&paramform=no $>$ Acesso em: 11 nov. $2011 \mathrm{~b}$.

ALMEIDA, J.E.M. Avaliação de fungos entomopatogênicos visando ao controle do cupim subterrâneo Heterotermestenuis (Hagen, 1858) (Isoptera, Rhinotermitidae). 1994. 105f. Dissertação (Mestrado em Entomologia) - Escola Superior de Agricultura "Luiz de Queiroz"/USP, Piracicaba.

ALMEIDA, J.E.M.; ALVES, S.B. Controle de Heterotermestenuis (Hagen) (Isoptera: Rhinotermitidae) em cana-de-açúcar com iscas Termitrap ${ }^{\circledR}$ associadas ao fungo entomopatogênicoBeauveriabassiana (Bals.) Vuill. e/ou inseticidas em época seca. Arquivos do Instituto Biológico, v.66, n.2, p.85-90, 1999.

ALMEIDA, J.E.M.; ALVES, S.B. Mortalidade de Heterotermestenuis (Hagen) atraídos por armadilhas com Beauveriabassiana (Bals.) Vuill. eimidacloprid. Anais da Sociedade Entomológica do Brasil... v.25, n.3, p.507-512, 1996.

ALVES, S.B. Fungos entomopatogênicos. In: ALVES, S.B. (ed.). Controle microbiano de insetos, 2. ed. Piracicaba: Fealq, 1998. p.289-381.

AZEREDO, E.H. de; CASSINO, P.C.R. Efeitos de duas formulações diferenciadas de thiamethoxam (Actara) na proteção de plantas de batata a pragas de solo e da parte aérea. Rev. Univ. Rural, Ser. Ciênc. da Vida, v.30, n.1, p.1-26, 2010. Disponível em: 〈http://www.editora.ufrrj.br/rcv2/vida_30_1/06v08.pdf > Acesso em: 20 nov. 2011.

BADILLA, F.F.; ALVES, S.B. Controle do gorgulho-da-cana-de-açúcar SphenophoruslevisVaurie, 1978 (Coleoptera: Curculionidae) com Beauveria sp. em condições de laboratório e campo. Anais da Sociedade Entomológica do Brasil, v.20, n.1, p.251-262, 1991.

CANINI, F.L.S. Manejo de Spodopterafrugiperda utilizando os fungos entomopatogênicosMetarhiziumanisopliae e Beauveriabassiana em milho. 2010. 40f. Monografia (Trabalho de Conclusão de Curso em Agronomia) - Centro Universitário Moura Lacerda, Ribeirão Preto.

CASTANHEIRA, R.S. Influência da adubação do milho (ZeamaysL.) sobre a eficiência da Bauveriabassiana, no controle da Spodopterafrugiperda. Ecossistema, v.18, p.119-129, 1993.

DANIELI, T. et al. Eficiência dos fungos Metarhiziumanisopliaee Beauveriabassiana aplicados em pulverização ou granulado no controle de Spodopterafrugiperda em milho "safrinha". In: SIMPÓSIO DE CONTROLE BIOLÓGICO, 11., Bento Gonçalves, 2009. Resumo... Porto Alegre: Unisinos, 2009. (CD$\mathrm{ROM})$

FARAG, N.A. Susceptibility of the cotton leafworm, Spodopteralittoralis 3(rd) instar larvae, to some bioinsecticides (Lepidoptera: Noctuidae). Egyptian Journal of Biological Pest Control, v.18, n.2, p.343$346,2008$. 
GOSSELIN, M.E.et al. Toxicity of spinosad and Beauveriabassiana to the black cutworm, and the additivity of subletal doses. Biocontrol Science and Technology, v.19, n.2, p.201-217, 2009. (doi: $10.1080 / 09583150802663285)$

GUAGLIUMI, P. Situação das cigarrinhas e das brocas nos canaviais do estado de Santa Catarina, e descobrimento de uma nova praga da cana. Brasil Açucareiro, v.81, n.3, p.10-13, 1973.

GUAGLIUMI, P.; MENDES, A.C. Três novas pragas da cana-de-açúcar no Estado de São Paulo. Brasil Açucareiro, v.82, n.2, p.80-81, 1974.

GUTIÉRREZ, L.S.; CARDENAS, W.H.K.; GUTIÉRREZ, G.S. Estudio bioeconómico de la utilización de Metarhiziumanisopliae junto con inhibidores de síntesis de quitina en el control del gusano cogollero en sorgo. Manejo Integrado de Plagas, n.36, p.1-6, 1995.

HENDERSON, C.F.; TILTON, E.W. Tests with acaricides against the brown wheat mite.Journal of Economic Entomology, v.48, p.157-161, 1955.

KAUR, G.; PADMAJA, V. Evaluation of Beauveria bassiana isolates for virulence against Spodoptera litura (Fab.)(Lepidoptera: Noctuidae) and their characterization by RAPD-PCR.African Journal of Microbiology Research, v.2, n.11, p.299-307, 2008.

KULKARNI, S.A. et al. Comparison of Metarhizium isolates for biocontrol of Helicoverpa armigera (Lepidoptera: Noctuidae) in chickpea. Biocontrol Science and Technology, v.18, n.8, p.809-828, 2008. (doi: 10.1080/09583150802366475)

LEVINE, E.; FELSOT, A. Effectiveness of acephate and carbofuran seed treatments to control the black cutworm, Agrotis ipsilon (Lepidoptera: Noctuidae), on field corn. Journal of Economic Entomology, v.78, n.6, p.1415-1420, 1985.

MACEDO, L.P.M.; GARCIA, J.F.; BOTELHO, P.S.M. Outros besouros-praga da cana-de-açúcar. In: PINTO, A. de S. (org.). Controle de pragas da cana-de-açúcar. Sertãozinho: Biocontrol, 2006. p.49-52.

MARTINS, G.L.M.et al. Efeito de alguns inseticidas sobre Spodoptera frugiperda (Lepidoptera: Noctuidae) e Dichelopssp. (Homoptera: Pentatomidae) na fase inicial da cultura do milho. Revista Científica Eletrônica de Agronomia, v.5, n.9, 2006. Disponível em: <http://www.revista.inf.br/agro09/artigos/ARTIGO02.pdf> Acesso em: 10 nov. 2010.

MARTINS, R.D. Manejo da lagarta-do-cartucho, Spodoptera frugiperda, com fungos entomopatogênicos em milho de segunda safra. 2010. 26f. Monografia (Trabalho de Conclusão de Curso em Agronomia) - Centro Universitário Moura Lacerda, Ribeirão Preto.

MENDONÇA, A.F.et al. Broca comum da cana-de-açúcar. In: MENDONÇA, A.F. (ed.). Pragas da cana-de-açúcar. Maceió: Insetos \& Cia, 1996. p. 49-82.

MICHEREFF, M.; TORRES, J.B.; ANDRADE, L.N.T.; NUNES, M.U.C. Effect of some biorational insecticides on Spodoptera eridania in organic cabbage.Pest Management Science, v.64, n.7, p.761-767, 2008. (doi: 10.1002/ps.1554)

OECO. Como andam os agrotóxicos no Brasil. Disponível em:

$<$ http://www.oeco.com.br/reportagens/25276-como-andam-os-agrotoxicos-no-brasil > Acesso em: 10 nov. 2011.

PARRA, J.R.P.; BOTELHO, P.S.M.; PINTO, A. de S. Controle biológico de pragas como um componente chave para a produção sustentável da cana-de-açúcar. In: CORTEZ, L.A.B. (Org.). Bioetanol de cana-de-açúcar: P\&D para produtividade e sustentabilidade. São Paulo: Blucher, 2010. p.441-450. 
PINTO, A. de S.; ANNIBAL, R.M.; ANIBAL, J.P. Concentração de conídios, forma e época de aplicação de Metarhizium anisopliae no controle de Spodoptera frugiperda (Lepidoptera: Noctuidae) em milho. In: CONGRESSO NACIONAL DE MILHO E SORGO, 25., Cuiabá, 2004. Resumo expandido... Sete Lagoas: Embrapa Milho e Sorgo, 2004. (CD-ROM)

PINTO, A. de S.; BOTELHO, P. S. M.; OLIVEIRA, H. N. de. Guia ilustrado de pragas e insetos benéficos da cana-de-açúcar. Piracicaba: CP 2, 2009. 160p.

PINTO, A. de S.; GARCIA, J. F.; OLIVEIRA, H. N. de. Manejo das principais pragas da cana-de-açúcar. In: SEGATO, S. V.et al. (orgs.). Atualização em produção de cana-de-açúcar. Piracicaba: CP 2, 2006. p.257-280.

PLANALSUCAR. Nova praga da cana-de-açúcar no Sul do Brasil Hyponeuma taltula(Schaus) (Lepidoptera: Noctuidae). In: INSTITUTO DO AÇÚCAR E DO ÁLCOOL (Ed.). Relatório Anual do Programa Nacional de Melhoramento da Cana-de-açúcar... Rio de Janeiro: Ministério da Indústria e Comércio, 1974. p.42.

RONDA,J.S.S. Inseticidas de origem microbiana e vegetal no controle de pragas do milho. In: CONGRESSO NACIONAL DE MILHO E SORGO, 25., Cuiabá, 2004. Resumos... Sete Lagoas: Embrapa-CNPMS, 2004. (CD-ROM)

SILVA, A.G.A. et al. Quarto catálogo dos insetos que vivem nas plantas do Brasil: seus parasitos e predadores. Rio de Janeiro: Ministério da Agricultura, 1968. 622p.

SILVEIRA, M. dos S.et al. Patogenicidade de Metarhizium anisopliae (Metsch.) Sorok. E Beauveria bassiana (Bals.) Vuill. sobre ovos de Diatraea saccharalis (Fabr.) de diferentes idades. In: SIMPÓSIO DE CONTROLE BIOLÓGICO, 10., Brasília, 2007. Anais... Brasília: Cenargen, 2007. (CD-ROM)

STATSOFT. Statistica for Windows: computer program manual. Tulsa, OK: StatSoft Inc., 1996.

UDOP - UNIÃO DOS PRODUTORES DE BIOENERGIA. Evolução da produtividade cana-deaçúcar safras 2005/2006 a 2010/2011. Disponível em:

$<$ http://www.udop.com.br/download/estatistica/area_cultivada/01set11_area_cultivada_produtivadade_bra sil.pdf> Acesso em: 12 nov. 2011.

VERCESI, D.B. et al. Eficiência dos fungos Metarhizium anisopliae e Beauveria bassiana aplicados em pulverização ou granulado no controle de Spodoptera frugiperda em milho "safrinha". In: CONGRESSO BRASILEIRO DE ENTOMOLOGIA, 22., Uberlândia, 2008. Anais... Sete Lagoas: UFU/Embrapa Milho e Sorgo/UFV, 2008. (CD-ROM)

WANG, S.C.; DAUTERMAN, W.C. Toxicity, penetration, pxcretion, and metabolism of carbofuran in larvae of the tobacco budworm and the corn earworm (Lepidoptera: Noctuidae). Journal of Economic Entomology, v.88, n.2, p.237-240, 1995.

ZENKER, M.M. et al. Caracterização morfológica dos imaturos de Hyponeuma taltula(Schaus) (Lepidoptera, Noctuidae, Herminiinae). Revista Brasileira de Zoologia, v.24, n.4, p.1101-1107, 2007. 